\title{
Pitfalls in Prostate Cancer Magnetic Resonance Imaging
}

\author{
Kuldeep Yadav ${ }^{1}$ Binit Sureka ${ }^{1}$ Poonam Elhence ${ }^{2}$ \\ ${ }^{1}$ Department of Diagnostic and Interventional Radiology, All India \\ Institute of Medical Sciences, Jodhpur, Rajasthan, India \\ 2Department of Pathology and Laboratory Medicine, All India \\ Institute of Medical Sciences, Jodhpur, Rajasthan, India \\ ${ }^{3}$ Department of Urology, All India Institute of Medical Sciences, \\ Jodhpur, Rajasthan, India
}

\author{
Gautam Ram Choudhary ${ }^{3}$ Himanshu Pandey ${ }^{3}$
}

Ind J Med Paediatr Oncol 2021;42:80-88.

\begin{abstract}
Address for correspondence Binit Sureka, Associate Professor, MD, DNB, MBA, Department of Diagnostic and Interventional Radiology, All India Institute of Medical Sciences, Jodhpur, Rajasthan, 342005, India (e-mail: binitsurekapgi@gmail.com).
\end{abstract}

\author{
Abstract \\ Keywords \\ - Cancer \\ - magnetic resonance \\ imaging \\ - pitfall \\ - prostate
}

Image-guided prostate biopsies are changing the outlook of prostate cancer (PCa) diagnosis, with the degree of suspicion on multiparametric magnetic resonance imaging (mp-MRI) being a strong predictor of targeted biopsy outcome. It is important not only to detect these suspicious lesions but also to be aware of the potential pitfalls in $\mathrm{mp}$-MRI prostate imaging. The aim of this pictorial essay is to show a wide spectrum of representative cases, which are frequently misdiagnosed as PIRADS $4 / 5$ while reporting $\mathrm{mp}-\mathrm{MRI}$ of the prostate. We provide some valuable recommendations to avoid these fallacies and improve mp-MRI of prostate evaluation.

\section{Introduction}

In the past few years, multiparametric magnetic resonance imaging (mp-MRI) of the prostate has undergone many technical advances, related to scientific research, in the diagnosis of prostate cancer (PCa). At present, mp-MRI prostate has become the imaging modality of choice for the detection, localization, staging and grading of $\mathrm{PCa}$, and response assessment to therapy. ${ }^{1,2} \mathrm{Mp}-\mathrm{MRI}$ has been proved to be an imaging modality superior to any other imaging technique in evaluating prostate pathologies for several reasons such as detailed anatomical and functional information of the prostate gland. The detection of PCa on T2-weighted imaging (T2WI) can be confounded by false-positive findings such as prostatitis, calcification, postbiopsy hemorrhage, benign prostatic hyperplasia, fibrosis, radiation, and hormonal tissue changes. The mp-MRI combines anatomic details with functional information while evaluating tissues by diffusion-weighted imaging (DWI) and perfusion dynamic contrast-enhanced (DCE) sequences. The multiparameter concept refers to the fact that magnetic resonance sequences in isolation have limited accuracy in detecting and classifying prostate neoplasms; however, a combination of several sequences has demonstrated high accuracy in the detection of PCa. ${ }^{3}$ The positivity rates for the biopsy is around 70 to $90 \%$ for MRI findings highly suspicious for PCa; however, true data regarding false-negative rates of MRI-transrectal ultrasound (TRUS) fusion-targeted biopsies are lacking. ${ }^{4}$ Predicting final histopathological Gleason score on mp-MRI still remains a challenge, which has not been taken into account with the current prostate imaging reporting and data system (PIRADS) scoring system. However, various models are used, mainly taking into consideration clinical factors such as age, preoperative prostate-specific antigen (PSA) level, PIRADS score $\geq$ 4 , and the number of positive biopsy cores involved with the cancer to predict the final Gleason score. ${ }^{5}$

The term "pitfall" means a hidden or unsuspected danger or difficulty. It is mandatory to know the pitfalls that can be encountered while reporting mp-MRI prostate. To standardize the reporting of mp-MRI prostate, the PIRADS was developed by the American College of Radiology and European
DOI https://doi.org/ 10.1055/s-0041-1730757 ISSN 0971-5851

\section{(c) 2021. Indian Society of Medical and Paediatric Oncology}

This is an open access article published by Thieme under the terms of the Creative Commons Attribution-NonDerivative-NonCommercial-License, permitting copying and reproduction so long as the original work is given appropriate credit. Contents may not be used for commercial purposes, or adapted, remixed, transformed or built upon. (https://creativecommons.org/licenses/by-nc-nd/4.0/).

Thieme Medical and Scientific Publishers Pvt. Ltd. A-12, 2nd Floor, Sector 2, Noida-201301 UP, India 
Society of Urogenital Radiology in 2012. At present, the PIRADS version 2.1 is effective since March 2019.

A sound knowledge of the pelvic anatomy and pathology, with emphasis on that of the prostate and PI-RADS v2.1 chalk out the "dominant" sequences needed to adequately evaluate the anatomical zones of the prostate, with emphasis on the utilization of diffusion/apparent diffusion coefficient (ADC) map for the peripheral zone (PZ) and T2 sequences for the central zone $(\mathrm{CZ}){ }^{3}$

This pictorial essay aims to give special importance to the cases of pitfalls of PCa, and suggestions are given to avoid them in daily clinical practice. Pitfalls can be divided into those conforming to the anatomical zones of the prostate gland, including the anterior fibromuscular stroma (AFMS), surgical capsule, posterior pseudolesion of the PZ (teardrop sign), periprostatic venous plexus, and neurovascular bundle, which can simulate cancer and benign conditions such as benign prostatic hyperplasia and inflammatory/infectious pathology, and other conditions that can mimic malignant lesions of prostate. Various pitfalls in prostate imaging are highlighted in - Table $\mathbf{1}$, and various MRI recommendations to avoid these pitfalls are tabulated in $\boldsymbol{- T a b l e ~} \mathbf{2}$.

\section{Magnetic Resonance Anatomy of Prostate}

Prostate gland consists of the base, the midgland, and the apex. It is divided into four histologic zones: (a) the AFMS which contains no glandular tissue; (b) the transition zone (TZ), surrounding the urethra proximal to the verumontanum, which contains $5 \%$ of the glandular tissue; (c) the $\mathrm{CZ}$, surrounding the ejaculatory ducts, which contains approximately $20 \%$ of the glandular tissue; and (d) the outer PZ, containing 70 to $80 \%$ of the glandular tissue ( - Fig. 1).

On mp-MRI, T1-weighted (T1W) images are used primarily to determine the presence of hemorrhage within the prostate and delineate the outline of the gland. T2W images are used to interpret zonal anatomy and abnormalities within the gland and evaluate extraprostatic invasion. On T2W images, clinically significant cancers in the PZ usually appear as round or ill-defined hypointense focal lesions. DWI reflects the random motion of water molecules and is a key component of the prostate mp-MRI examination. It should include an ADC map and high b-value images.

Most clinically significant cancers have restricted/impeded diffusion compared with normal tissues and thus appear hypointense on ADC maps. DCE MRI is defined as the acquisition of rapid T1W gradient echo scans before, during, and after the intravenous (IV) administration of a low-molecular weight gadolinium-based contrast agent. As with many other malignancies following a bolus injection of contrast, PCas often demonstrate early enhancement compared with normal tissue.

\section{Anatomic Pitfalls}

\section{Hypertrophic Anterior Fibromuscular Stroma}

According to the McNeal zonal anatomy distinction, AFMS is the most anterior portion of the gland, situated between
Table 1 Pitfalls in mp-MRI prostate imaging

\begin{tabular}{|c|c|}
\hline MR pitfalls & Entity and signs \\
\hline \multirow[t]{4}{*}{ Anatomic } & Hypertrophic fibromuscular stroma \\
\hline & Surgical capsule \\
\hline & Periprostatic venous plexus \\
\hline & Neurovascular bundle \\
\hline \multirow[t]{10}{*}{ Benign pathologies } & Moustache sign \\
\hline & Moustache-like sign \\
\hline & Teardrop sign \\
\hline & Teardrop-like sign \\
\hline & Ectopic/extruded BPH nodule \\
\hline & Prostatitis \\
\hline & Pitfall versus pitfall \\
\hline & PCa in moustache sign \\
\hline & $\mathrm{PCa}$ in median posterior change \\
\hline & Ectopic BPH nodule or abscess \\
\hline latrogenic changes & Postbiopsy hemorrhage \\
\hline \multirow[t]{5}{*}{ Posttreatment changes } & Residual prostate gland \\
\hline & Fibrosis \\
\hline & Granulation tissue \\
\hline & Hemorrhage \\
\hline & Brachytherapy seeds \\
\hline \multirow[t]{3}{*}{ Artifacts } & Malpositioned endorectal coil \\
\hline & Postbiopsy changes \\
\hline & Foley's catheter \\
\hline \multirow[t]{2}{*}{ Others } & Calcifications \\
\hline & Periprostatic lymph nodes \\
\hline
\end{tabular}

Abbreviations: $\mathrm{BPH}$, benign prostatic hyperplasia; $\mathrm{PCa}$, prostate cancer; $\mathrm{mp}-\mathrm{MRI}$, multiparametric magnetic resonance; MR, magnetic resonance.

the two lobes that constitute the TZ, which often appears to be flattened by them. ${ }^{6}$ This zone corresponds to a band of tissue formed by muscle cells and connective tissue, which is devoid of glandular tissue. There is variable prominence of AFMS among individuals. Due to the compressive effect of benign prostatic hyperplasia (BPH), it is less prominent in men with increased age and gland size. ${ }^{7}$ Owing to its histological composition (muscle cells and connective tissue), it appears homogeneously hypointense on $\mathrm{T} 2 \mathrm{~W}$ images, which can be mistaken for PCa and also tend to be hypointense (-Fig. 2). In some cases where hypertrophy of AFMS may mimic PCa on T2W images, signal characteristics on DWI and enhancement on DCE-MRI should be correlated. ${ }^{8}$

\section{Surgical Capsule}

The surgical capsule referred to the connective tissue support that is concentric fibrous tissue normally found separating the $\mathrm{TZ}$ and the $\mathrm{PZ}$ of the prostate. Due to its fibrous tissue composition, it shows low-signal intensity on T2W images. Normally, it is a linear structure, without a nodular or 
Table 2 Fallacies and recommendations to avoid pitfalls

\begin{tabular}{|l|l|}
\hline Pitfall & $\begin{array}{l}\text { Key MR sequence to avoid } \\
\text { pitfall }\end{array}$ \\
\hline $\begin{array}{l}\text { Hypertrophic } \\
\text { fibromuscular }\end{array}$ & DWI and DCE-MR sequence \\
\hline stroma & Multiplanar T2WI and DCE-MR \\
\hline Surgical capsule & Characteristic location, T2WI and \\
\hline Periprostatic venous plexus & DCE-MR \\
\hline & Characteristic location and \\
\hline Neurovascular bundle & Multiplanar T2WI \\
\hline & Coronal T2WI \\
\hline Moustache sign & Multiplanar T2WI and DWI \\
\hline Moustache-like sign & Coronal T2WI \\
\hline Teardrop sign & Multiplanar T2WI and DWI \\
\hline Teardrop-like sign & Multiplanar T2WI and DWI \\
\hline Ectopic/extruded BPH & \\
\hline nodule & Clinical history correlation and \\
\hline Prostatitis & DCE-MR \\
\hline & TI-WI and DCE-MR \\
\hline Postbiopsy hemorrhage & DWI and DCE-MR \\
\hline Fibrosis & DWI \\
\hline Granulation tissue & DCE-MR and DWI \\
\hline Brachytherapy seeds & \\
\hline Abbrevtons: DWI, & \\
\hline
\end{tabular}

Abbreviations: DWI, diffusion-weighted imaging, DCE, dynamic contrast-enhanced; MR, magnetic resonance; T2WI, T2-weighted image; T1WI, T1-weighted image.

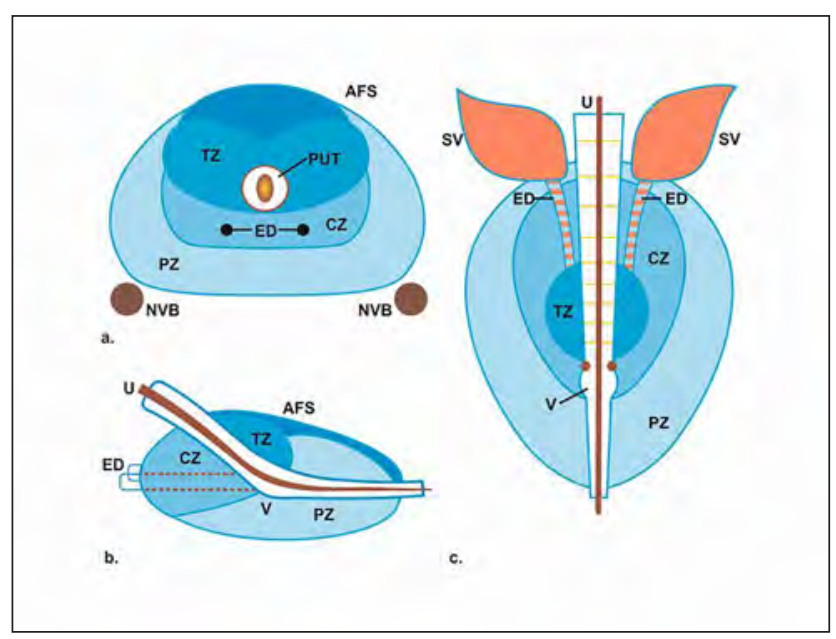

Fig. 1 Line diagram in different planes showing normal anatomy of prostate (AFS-anterior fibromuscular stroma, TZ-transitional zone, CZ-central zone; PZ-peripheral zone; ED-ejaculatory ducts; PUTprostatic urethra; NVB-neurovascular bundle; SV-seminal vesicles; U-urethra; V-verumontanum.

mass-like configuration ( - Fig. 3 ). In some cases, asymmetric thickening of the capsule may simulate a suspicious lesion. Multiplanar, high-resolution T2W images and DCE-MRI help us to differentiate it from the malignant lesion. ${ }^{9,10}$

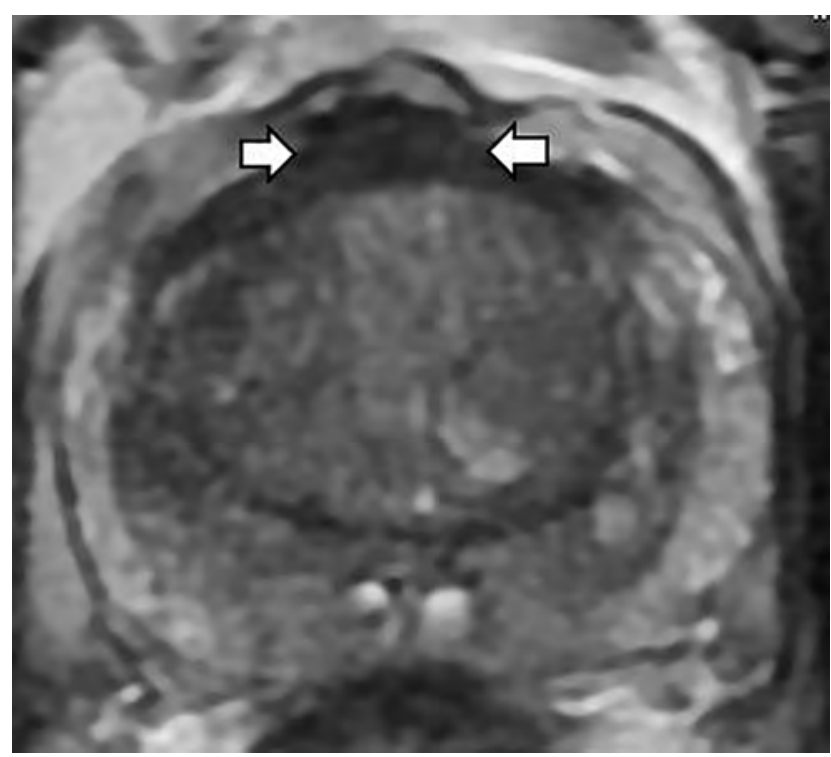

Fig. 2 Axial T2-weighted imaging (T2WI) showing triangular area of T2-hypointensity (arrows) in midlower prostate, corresponding to hypertrophic anterior fibromuscular stroma (AFS).

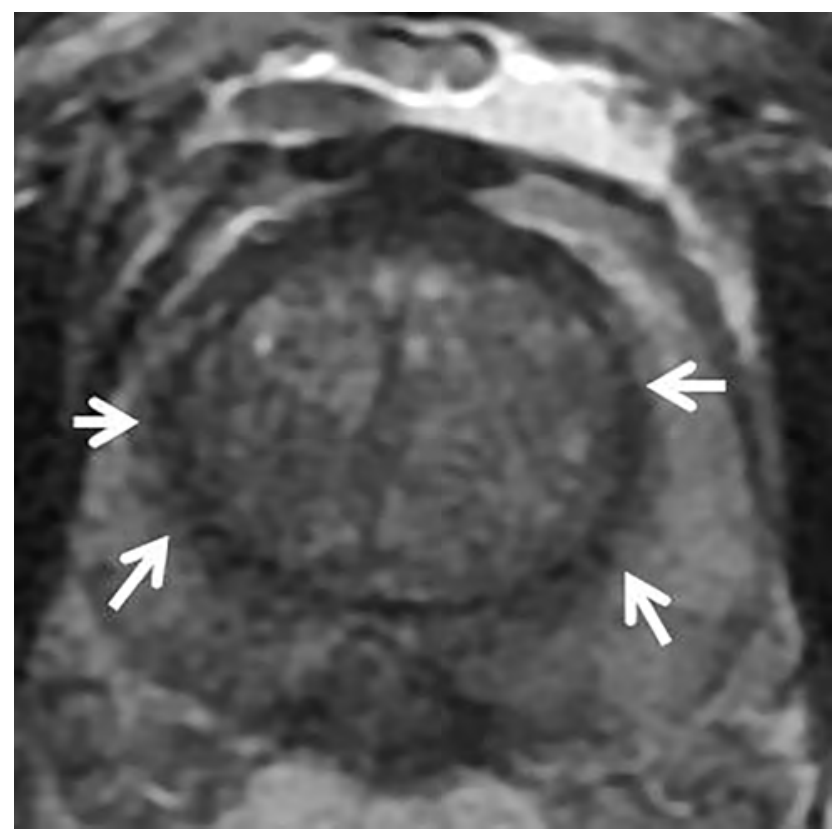

Fig. 3 Axial T2-weighted imaging (T2WI) showing T2-hypointense linear thickening (arrows) of surgical capsule, bulging into the bright peripheral zone (PZ).

\section{Periprostatic Venous Plexus}

The location of the periprostatic venous plexus at the anterolateral margin of the prostate, near the prostate capsule, presents a challenge in assessing lesions of the PZ. Also known as Santorini's plexus, it is in close proximity to the prostate capsule, and the size of the vessels is variable among patients. ${ }^{11}$ Patients with local inflammatory processes often show asymmetric dilation of the venous plexus. It appears rounded on axial images, with variable signal intensity on T2W images (depending on the blood flow inside the 


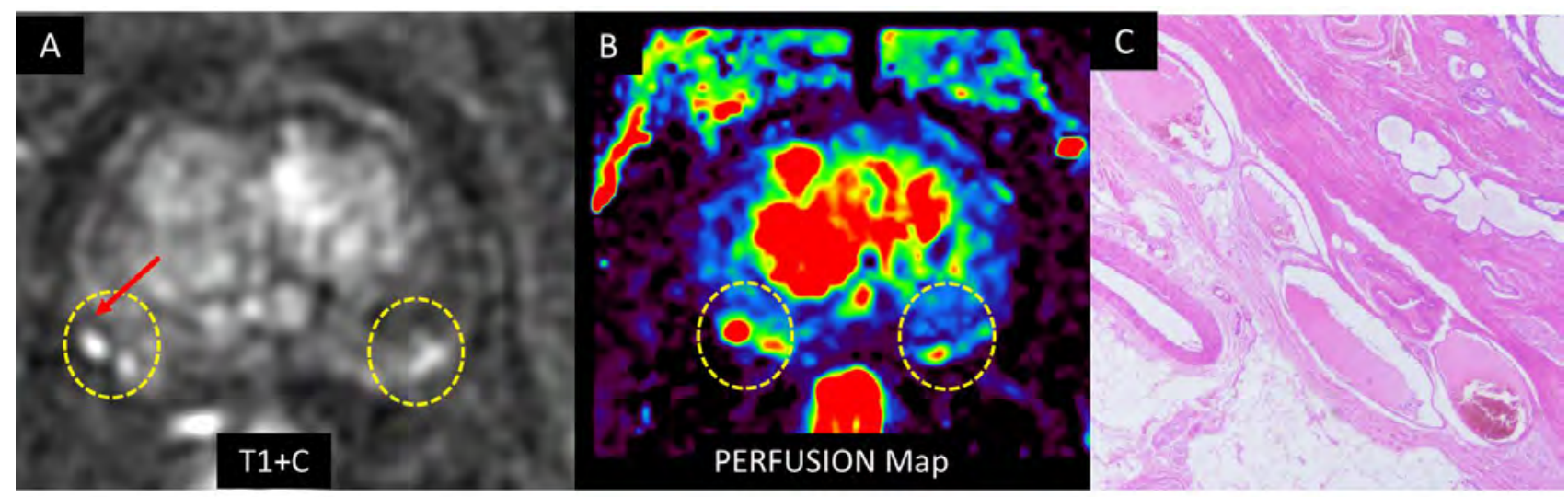

Fig. 4 Axial T1-weighted (T1W) dynamic contrast-enhanced MR images (a) showing dot like enhancement in the periphery of the prostate, (b) corresponding to perfusion map images showing increased perfusion suggestive of periprostatic venous plexus.

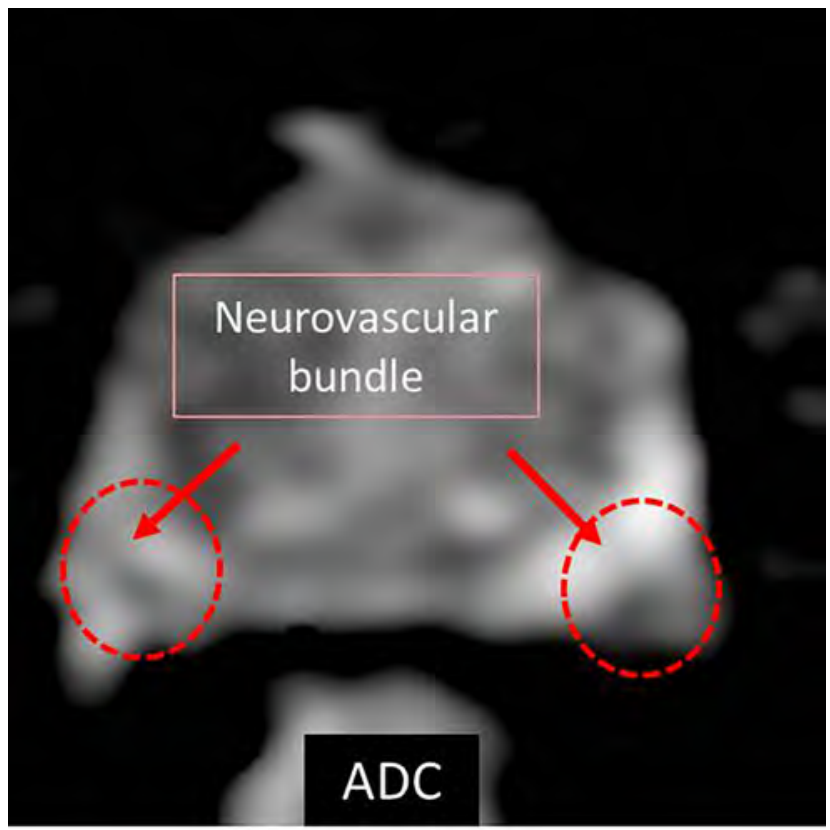

Fig. 5 Apparent diffusion coefficient (ADC) map showing hypointense dots in posterolateral region of prostate at 5 to 7 o' clock position, which could be erroneously diagnosed as peripheral zone (PZ) cancer, but location of these hypointense dots clinches the diagnosis of neurovascular bundle. vessel and flow voids) and low value on ADC map ( - Fig. 4). To avoid this pitfall, we must know the location of the periprostatic venous plexus, which is found in the anterolateral aspect of the PZ.

\section{Neurovascular Bundle}

The neurovascular bundle that innervates the corpora cavernosa is located at the posterolateral margin of the prostate, near the capsule, usually at 5 and 7 o'clock position. The preservation of this plexus is critical in postprostatectomy sexual function; however, in infiltrative PCa patients, surgeons are bounded to perform a wider resection. The neurovascular bundle may show a rounded appearance on axial images, with a low signal on T2W and ADC maps (-Fig. 5). In distorted ADC images, the neurovascular bundle may appear to be in the $\mathrm{PZ}$ and mimic a nodule. It is mandatory to evaluate these suspicious nodules on multiplanar, high-resolution T2W images.

\section{Benign Prostatic Hyperplasia}

\section{Moustache Sign (Bilateral Hyperplasia with Compression of Peripheral Zone)}

It is the presence of bilateral symmetrical small adenomatous nodules in the $\mathrm{TZ}$, which determines compression of the peripheral and $\mathrm{CZ}$ at the base/ middle of the prostate gland.

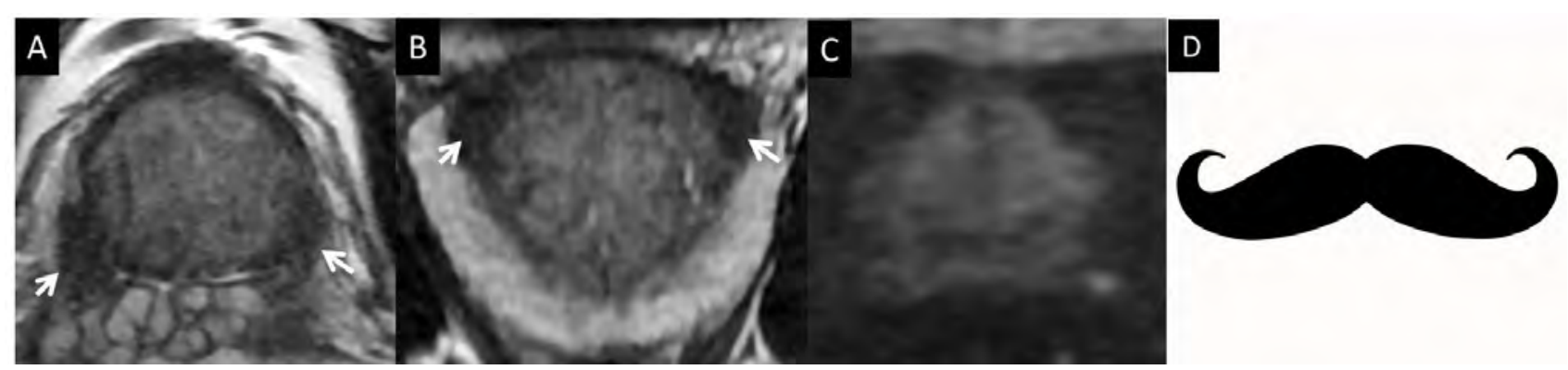

Fig. 6 Axial and coronal T2-weighted image (T2WI) of the prostate base (a and $\mathbf{b}$ ) showing bilateral symmetrical oval-shaped areas of low-signal intensity on T2WI (arrows) at the base of the prostate lateral to the opening of ejaculatory ducts. (c) No diffusion restriction is seen in these nodules. (d) Line diagram showing resemblance with a moustache. 


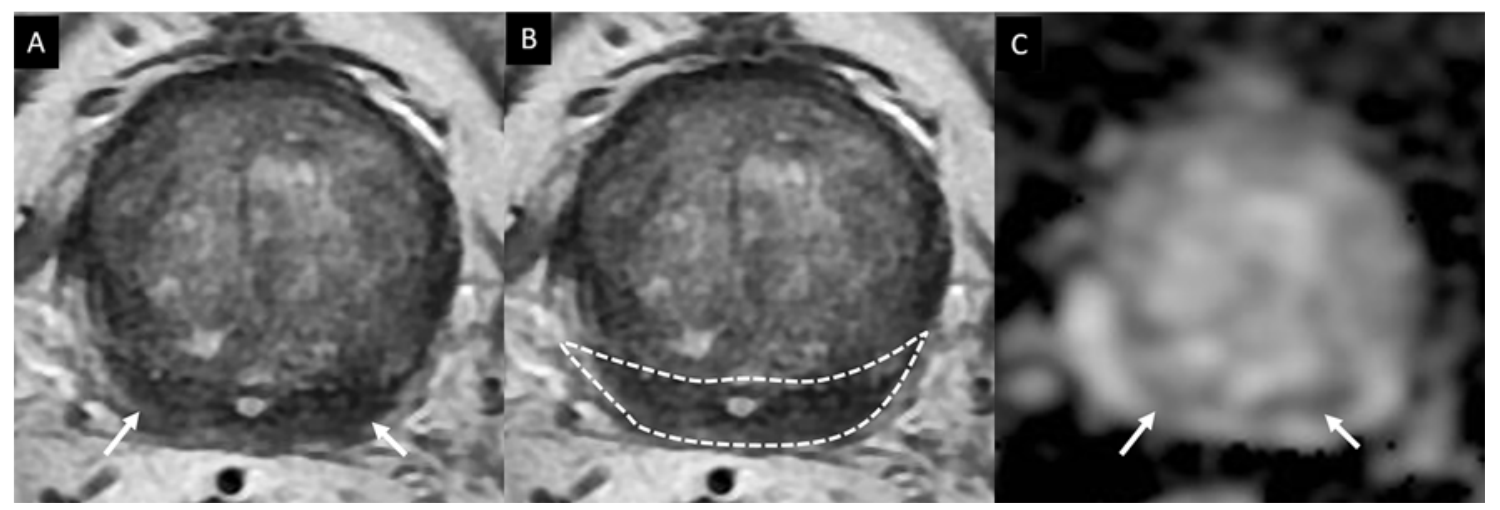

Fig. 7 Axial T2-weighted image (T2WI) of the prostate base, (a) showing a large adenoma bulging into the peripheral zone (PZ) (arrows) and (b) corresponding line diagram showing resemblance to a moustache, (c) with restricted diffusion in the apparent diffusion coefficient (ADC) map images.

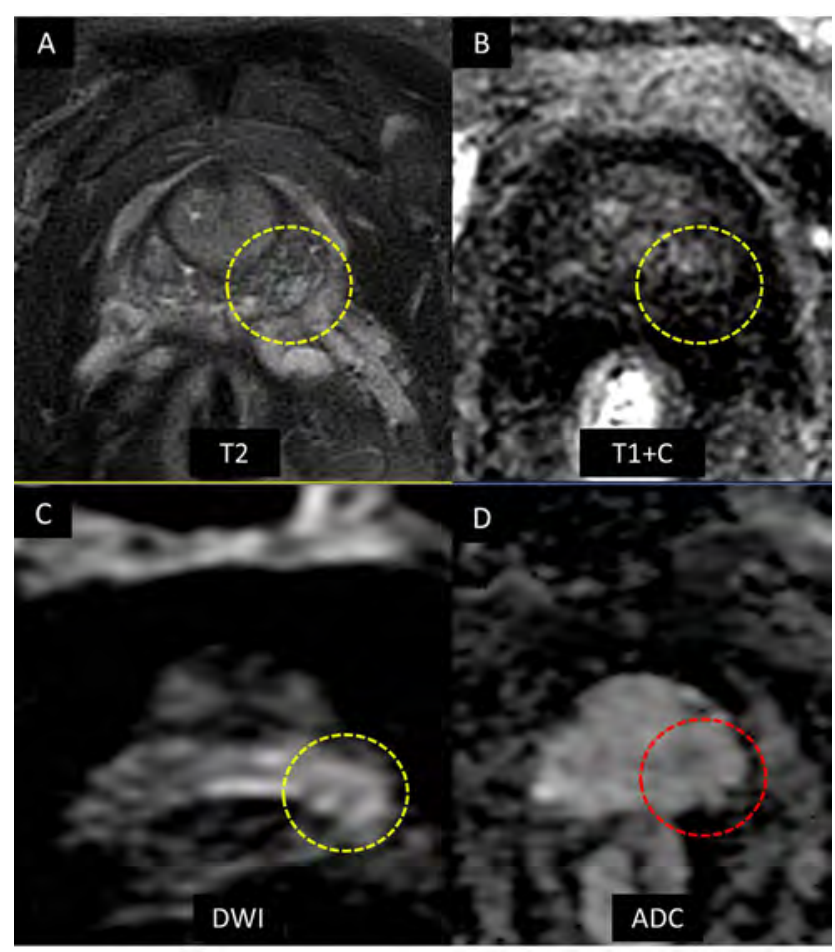

Fig. 8 MRI prostate showing asymmetrical oval area of low signal on T2-weighted imaging (T2WI) (a) depicting early enhancement on dynamic contrast-enhanced MRI (b), and diffusion restriction (c and d) in keeping with prostate cancer.
It is also referred to as "moustache sign", which is the presence of bilateral symmetrical homogenous oval-shaped areas of low-signal intensity on T2W images at the base of prostate, lateral to the opening of ejaculatory ducts, which appears like moustache ( - Fig. $\mathbf{6})$. Presence of symmetry is a crucial feature to determine whether you are dealing with a true "moustache sign" or a clinically significant suspicious lesion in this same area, especially when evaluating on coronal $\mathrm{T} 2$ sequence. $^{12}$

\section{Moustache-Like Sign (Larger Adenoma Against the Peripheral Zone)}

Protrusion of large adenomatous nodules in the PZ at the base of the gland shows moustache sign-like appearance ( - Fig. 7 ).

\section{Cancer in Moustache Sign}

The key feature to differentiate a pitfall from PCa is the symmetry of the finding. Asymmetry in the signal intensity with marked T2 hypointensity together with significant early focal enhancement and high grade of diffusion restriction points toward a malignant change-cancer in moustache sign (-Fig. 8).

\section{Teardrop Sign (Median Posterior Compressed Central Zone)}

This corresponds to the fusion and thickening of the prostate-fascia capsule in the midline at the junction of both

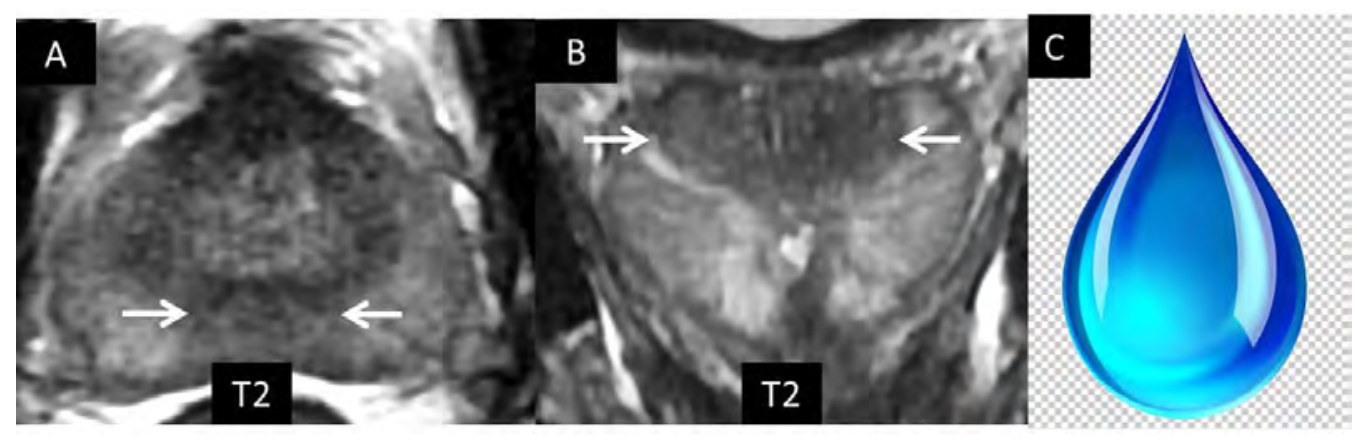

Fig. 9 (a) Axial T2-weighted imaging (T2WI) showing triangular area of hypointensity (arrows) in the peripheral zone (PZ) in the prostate base. (b) Coronal T2WI showing teardrop configuration (arrows) of the compressed central zone (CZ) between the transitional zone (TZ) and PZ. (c) Line diagram showing resemblance with a teardrop. 
the lobes surrounding the ejaculatory ducts, also called "teardrop sign" because of its shape that resembles a teardrop. This pseudolesion is found as a focal nodular hypointense area in the midline at the level of the base-middle third of the gland, as the $\mathrm{CZ}$ is compressed between the TZ and PZ (- Fig. 9). ${ }^{13}$

\section{Teardrop-like Sign (Protruding Benign Prostatic Hyperplasia Above the Verumontanum)}

Verumontanum is an important landmark in distinguishing compressed $\mathrm{CZ}$ and protruding $\mathrm{BPH}$ nodule. Teardrop sign is due to bulging of $\mathrm{CZ}$ above the verumontanum, while teardrop-like sign is due to bulging of $\mathrm{BPH}$ nodule above the verumontanum. ${ }^{14}$

\section{Ectopic/Extruded Benign Prostatic Hyperplasia Nodule} Ectopic adenomatous nodules are frequently misdiagnosed by inexperienced radiologists, interpreting it as mp-MRI prostate. These can mimic malignant lesions due to their rounded contour with low signal on T2WI; in some cases, these can show diffusion restriction with early enhancement pattern in perfusion study (DCE). Quantitative analysis of ADC values could improve the diagnostic accuracy in differentiating ectopic BPH nodule from peripheral PCa: the higher the ADC values, the higher the likelihood of ectopic BPH nodule. The crucial step in identification of these nodules is the demonstration of well-defined T2 hypointense capsule. Some of the ectopic BPH nodules may show tiny T2 hyperintense spots, corresponding to dilated acini, and this is a feature that can be used to distinguish them from PZ PCa (-Fig. 10).

\section{Bacterial Prostatitis (Acute and Chronic)}

It comprises the most common category of benign pathologies mimicking PCa. Acute bacterial prostatitis commonly affects young men; however, chronic prostatitis is commonly seen in

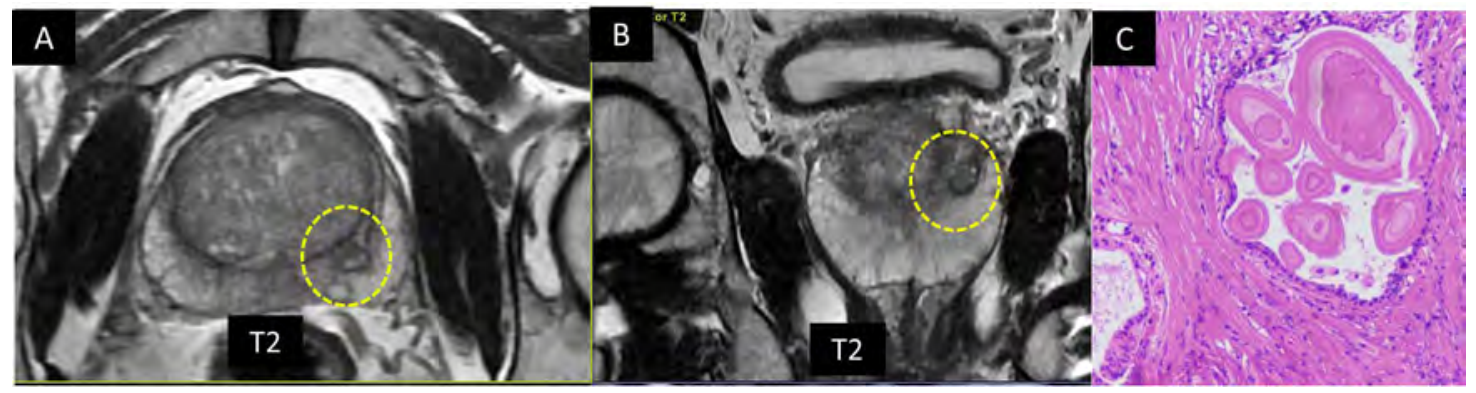

Fig. 10 Multiparametric MRI (mp-MRI) prostate showing well-defined encapsulated oval area of T2-hypointensity with internal bright spots within the nodule on axial and coronal T2-weighted imaging (T2WI) (a and b). Histopathology (c) showing stromal nodule with slit-like vessels, $\mathrm{H}$ and $\mathrm{E}, \times 2$ consistent with benign prostatic hyperplasia $(\mathrm{BPH})$ nodule.

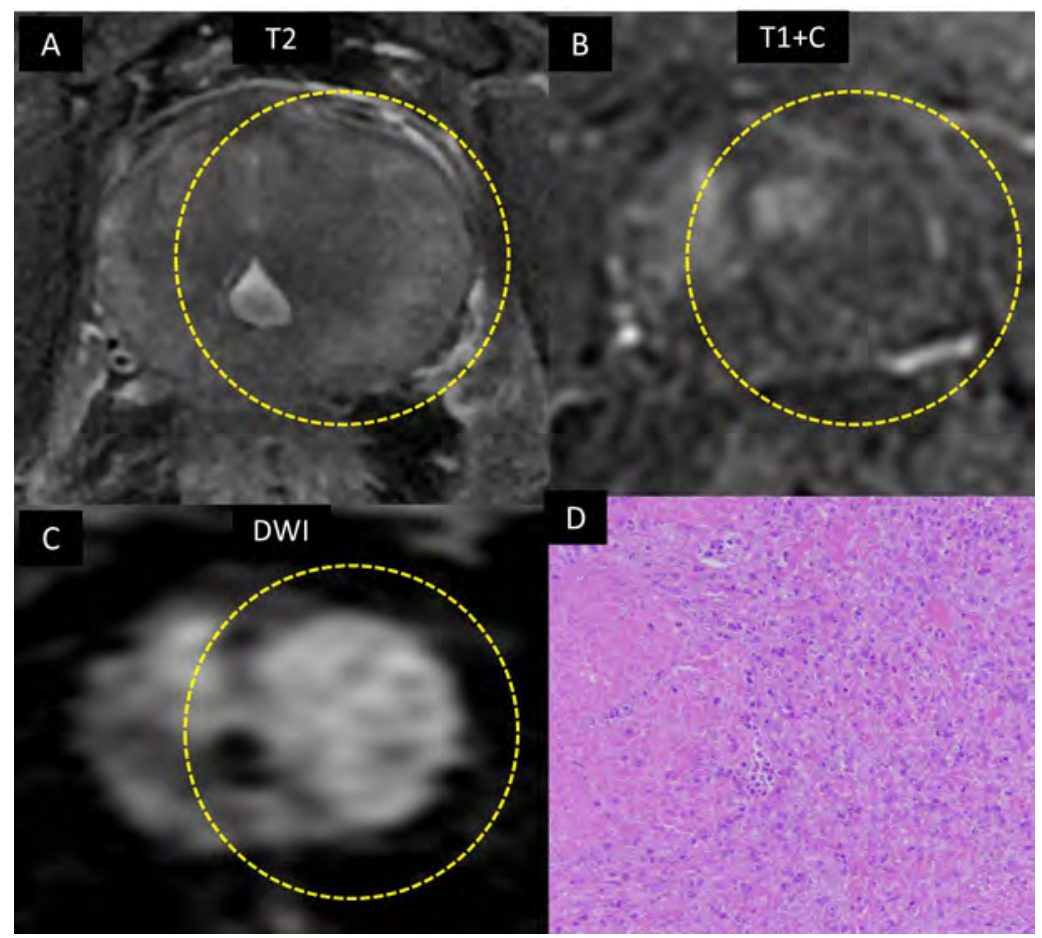

Fig. 11 Multiparametric MRI (mp-MRI) prostate showing altered area of signal with fuzzy margins and bulging contour (a), patchy enhancement on postcontrast images (b), and diffusion restriction (c). Histopathological images (d) depicting acute prostatitis with karyorrhectic debris and necrosis, $\mathrm{H}$ and $\mathrm{E}, \times 10$. 
older patients. Chronic prostatitis tends to be more indolent, and patients are often asymptomatic; however, acute prostatitis patients presents with fever, urinary tract symptoms, and often frequently associated with abscess. ${ }^{15}$ Although prostatitis reveals similar signal characteristics as PCa on mp-MRI, the morphology and degree of altered signal intensity with quantitative analysis of perfusion kinetics provide a clue to clinch the diagnosis. Inflammatory lesions exhibit nonmass-like morphology with ill-defined linear rather than rounded margins and diffuse lobar distribution ( - Fig. 11) ${ }^{16}$

\section{Granulomatous Prostatitis}

Most of the cases are idiopathic; however, prior history of intravesical bacillus Calmette-Guerin (BCG) therapy of the urinary bladder, tuberculous prostatitis, and transurethral resection of the prostate is frequently encountered. On

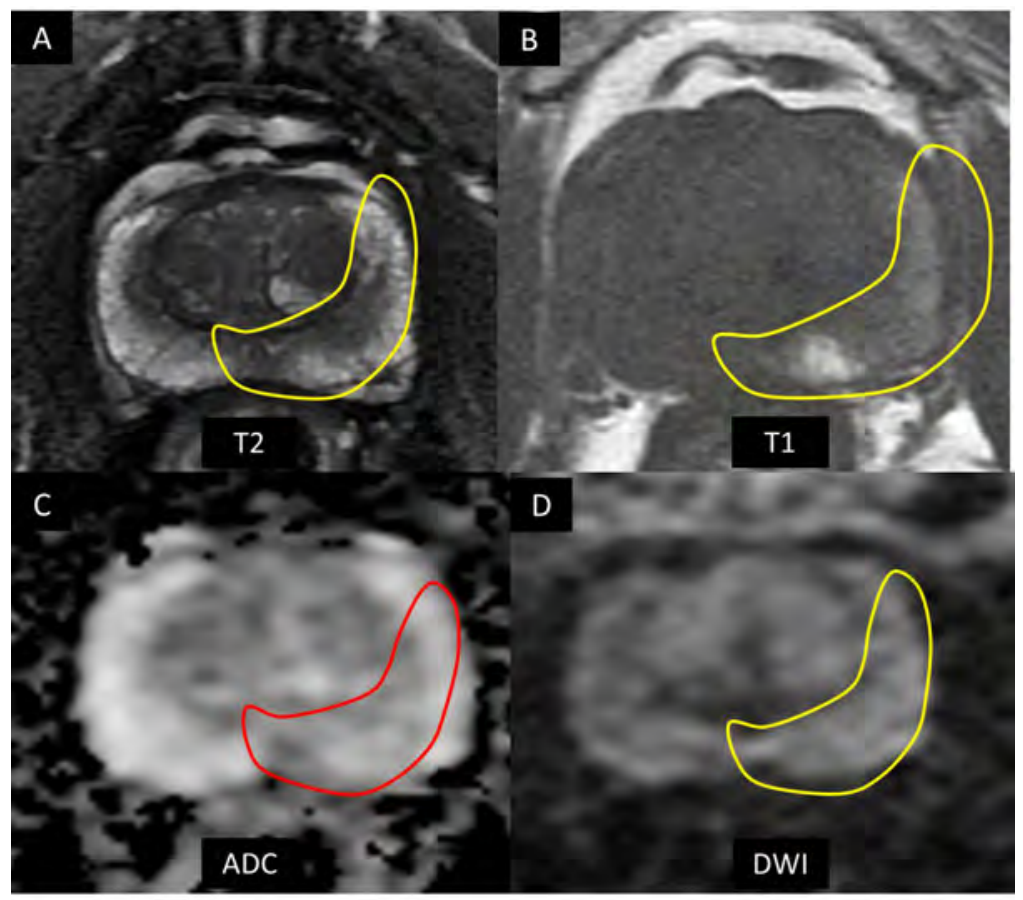

Fig. 12 MRI prostate showing an area of mild, low-signal intensity on T2-weighted imaging (T2WI) (a), with corresponding hyperintense area on precontrast T1-weighted imaging (T1WI) (b). Mild restricted diffusion on diffusion-weighted imaging (DWI) and apparent diffusion coefficient (ADC) maps is seen (c and $\mathbf{d}$ ). This is consistent with the products from the hemoglobin degradation after biopsy.

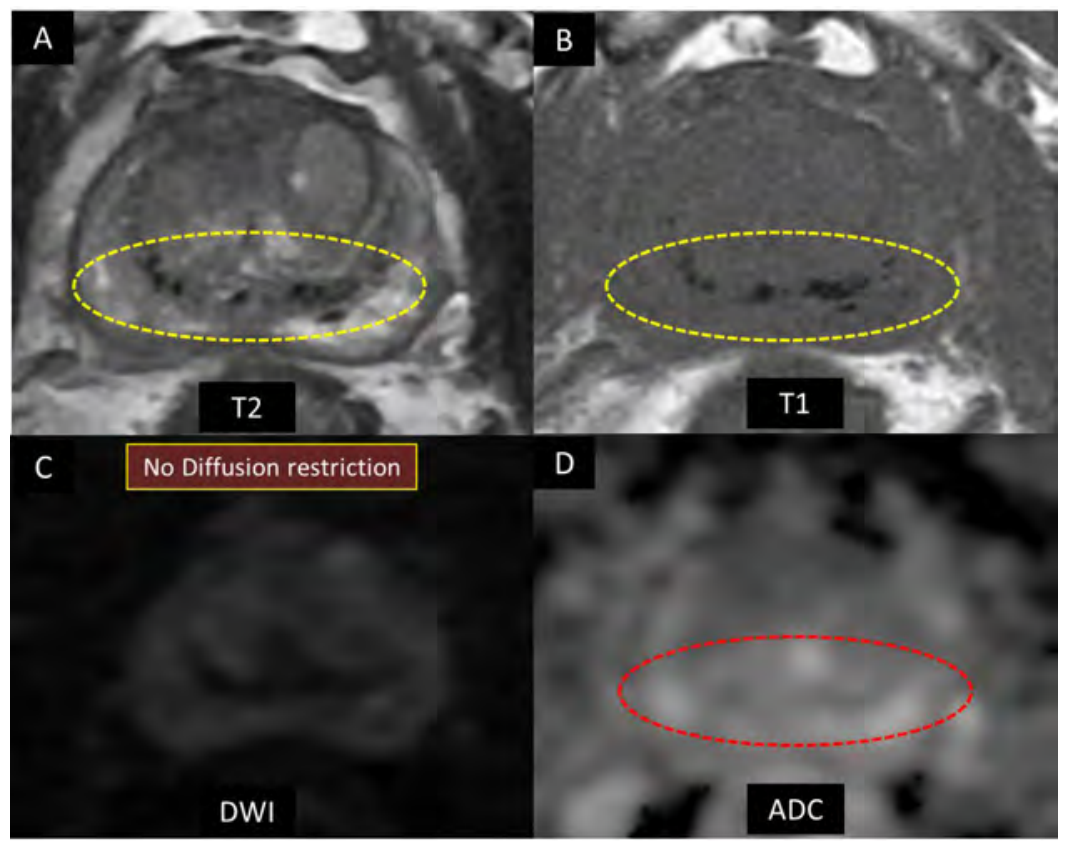

Fig. 13 MRI prostate showing hypointense foci on T2-weighted imaging (T2WI) (a), T1-weighted imaging (T1WI) (b), without any diffusion restriction on diffusion-weighted imaging (DWI) and apparent diffusion coefficient (ADC) maps (c and d), suggestive of calcification within the prostate gland. 


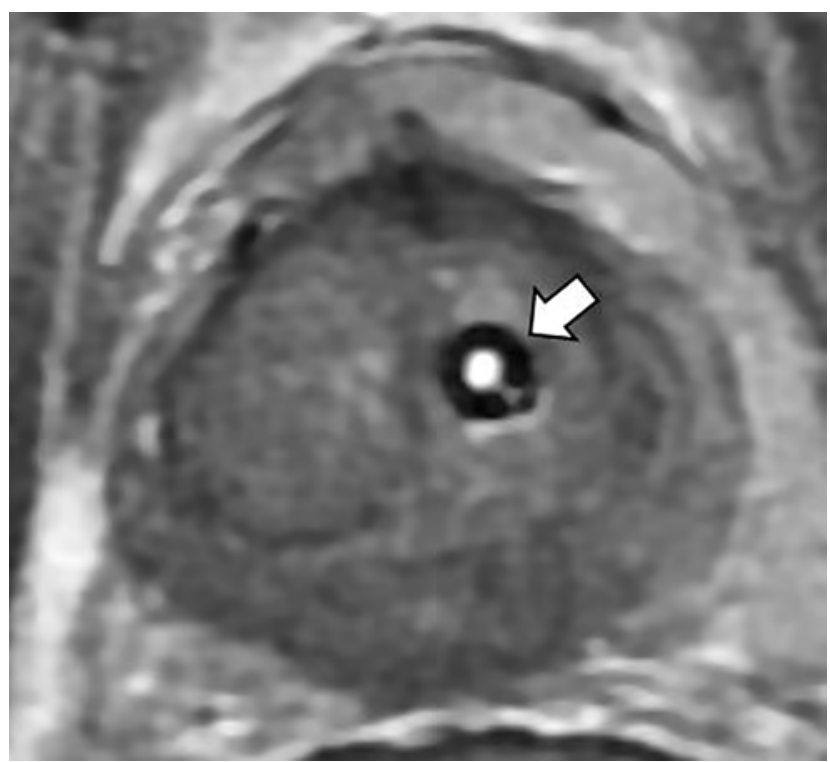

Fig. 14 Axial T2-weighted imaging (T2WI) showing artifact (arrow) due to Foley's catheter within the prostatic tissue.

mp-MRI, it may appear as mild-to-markedly $\mathrm{T} 2$ hypointense lesion, which shows diffusion restriction and early enhancement on DCE-MRI. ${ }^{17,18}$ After antimicrobial therapy, short-term follow-up MRI may be helpful to assess therapeutic response. ${ }^{9}$

\section{latrogenic Changes}

Biopsy needle-induced damage may determine a transient irregularity of the periprostatic capsule, leading to an overestimation of extraprostatic tumor extension.

\section{Postbiopsy Hemorrhage}

Hemorrhage is seen more frequently in the PZ than TZ. ${ }^{19}$

The signal intensity of postbiopsy hemorrhage depends on the state of hemoglobin degradation. In some metabolic states, it can mimic the suspicious lesion due to low signal on T2W and ADC images; however, it characteristically shows a high signal on the T1W sequence (-Fig. 12). As most of the mp-MRI studies are performed before prostate biopsies, the incidence of this pitfall is low, and if it is performed later, most of the experienced radiologists' advice to wait for at least 6 to 8 weeks postbiopsy. This delay is more advisable if it is required for staging purpose, as staging is more dependent on T2WI. If the primary aim is a diagnosis, then this delay can be avoided, as the likelihood of clinically significant $\mathrm{PCa}$ at the site of postbiopsy hemorrhage without a corresponding suspicious finding on MRI is low.

\section{Posttreatment Changes}

Treatment commonly includes radical prostatectomy, prostate-sparing focal therapy, or radiation therapy (RT), which can include external-beam RT or brachytherapy. ${ }^{20}$ There are some common mimics that should be identified in posttreatment cases. Granulation tissue and hemorrhage both will show early enhancement on DCE phase; however, on DWI, they will appear benign with no
Table 3 Key points to avoid pitfall in prostate imaging

The normal CZ appears homogeneously hypointense on T2WIs and dark on ADC maps in patients aged $42-84$ years. With these characteristics, it can mimic prostate cancer.

A symmetric appearance of the $C Z$ and its expected location can be helpful to differentiate it from PCa, but in $20 \%$ of cases, the CZ may be asymmetric.

Homogeneous low-signal intensity (erased charcoal sign), ill-defined margins, lack of capsule, lenticular shape, and invasion of the AFS are the MR imaging findings suggestive of TZ cancer on T2Wls.

Stromal hyperplasia tends to be well-defined, with a rounded encapsulated appearance.

A clinical history of urinary and/or sexual symptoms, fluctuating PSA level, or PSA response to antibiotics can alert the radiologist and the clinician to the possibility of prostatitis.

All the MR sequences must be corelated before reaching a diagnosis.

The pitfalls must be carefully addressed, and biopsy advised wherever required.

Abbreviations: ADC, apparent diffusion coefficient; AFS, anterior fibromuscular stroma; CZ, central zone; PCa, prostrate cancer; PSA, prostrate-specific antigen; T2WI, T2-weighted imaging; TZ, transition zone.

notable signal abnormalities. Posttreatment appears more hypointense than a recurrent tumor with the absence of early enhancement and presence of delayed enhancement during the venous phase. ${ }^{20}$ If metallic clips are used during surgery, the DCE sequence is more reliable than DWI. Early enhancement in DCE is highly suggestive of recurrence. All these changes should be correlated with clinical setting, type of treatment received, and PSA levels. ${ }^{21}$

\section{Artifacts}

\section{Mispositioned Endorectal Coil}

Use of endorectal is mandatory for the good-quality MRI scanning of prostate on 1.5T scanner. Accurate positioning of a coil is essential; otherwise, it creates artifacts affecting image quality, thus making the diagnosis difficult. Sometimes, it involves adjacent PZ and mimics PCa.

\section{Calcifications}

Incidence of prostate calcification is high and found basically due to concreted secretions or corpora amylacea..$^{22}$ They are generally detected in the TZ (central gland). Most of the time, they are indolent. They are characteristically hypointense in all sequences like T1, T2, and ADC, with blooming on susceptibility-weighted imaging (SWI), and do not show diffusion restriction ( - Fig. 13). CT is helpful to demonstrate the calcification.

\section{Foley's Catheter Tip}

Foley's catheter tip is hypointense on all sequences ( - Fig. 14). Coronal images will show its entire urethral course and its extension into the urinary bladder.

\section{Periprostatic Lymph Nodes}

Periprostatic lymph nodes are encountered rarely while interpreting mp-MRI prostate. These are commonly located 
at the base of the prostate gland laterally or posterolaterally. On mp-MRI imaging, these show well-defined rounded morphology with a hypointense signal on T2W sequence and marked diffusion restriction; however, the degree of diffusion restriction does not suggest metastatic involvement. Sometimes on low-resolution DWI/ADC images, periprostatic lymph nodes appeared to be intraprostatic in location. Detailed evaluation on high-resolution, multiplanar T2W images reveals its periprostatic location. ${ }^{11,23}$

\section{Conclusion}

All oncologists and radiologists involved in treating and interpreting mp-MRI prostate should be aware of these fallacies described above. Key points to avoid these pitfalls are illustrated in - Table 3. Despite the pitfalls described above, biopsy should be considered in men with an equivocal MRI and a PSA density of $>0.15$.

\section{Financial Support and Sponsorship} Nil.

\section{Conflicts of Interest}

There are no conflicts of interest.

\section{References}

1 Stabile A, Giganti F, Emberton M, Moore CM. MRI in prostate cancer diagnosis: do we need to add standard sampling? A review of the last 5 years. Prostate Cancer Prostatic Dis 2018;21(4):473-487

2 Ahmed HU, El-Shater Bosaily A, Brown LC, et al; PROMIS study group. Diagnostic accuracy of multi-parametric MRI and TRUS biopsy in prostate cancer (PROMIS): a paired validating confirmatory study. Lancet 2017;389(10071) :815-822

3 Turkbey B, Rosenkrantz AB, Haider MA, et al. Prostate imaging reporting and data system Version 2.1: 2019 update of prostate imaging reporting and data system Version 2. Eur Urol 2019;76(3):340-351

4 Costa DN, Kay FU, Pedrosa I, et al. An initial negative round of targeted biopsies in men with highly suspicious multiparametric magnetic resonance findings does not exclude clinically significant prostate cancer-Preliminary experience. Urol Oncol 2017;35(4):149.e15-149.e21

5 Alqahtani S, Wei C, Zhang Y, et al. Prediction of prostate cancer Gleason score upgrading from biopsy to radical prostatectomy using pre-biopsy multiparametric MRI PIRADS scoring system. Sci Rep 2020;10(1):7722
6 McNeal JE. The zonal anatomy of the prostate. Prostate 1981;2(1):35-49

7 Allen KS, Kressel HY, Arger PH, Pollack HM. Age-related changes of the prostate: evaluation by MR imaging. AJR Am J Roentgenol 1989;152(1):77-81

8 Labra WA, Zúñiga GÁ. Pitfalls in multiparametric prostate MRI. Rev Chil Radiol 2019;25:128-140

9 Rosenkrantz AB, Taneja SS. Radiologist, be aware: ten pitfalls that confound the interpretation of multiparametric prostate MRI. AJR Am J Roentgenol 2014;202(1):109-120

10 Semple JE. Surgical capsule of the benign enlargement of the prostate. Its development and action. BMJ 1963;1(5346) :1640-1643

11 Kitzing YX, Prando A, Varol C, Karczmar GS, Maclean F, Oto A. Benign conditions that mimic prostate carcinoma: MR imaging features with histopathologic correlation. Radiographics 2016;36(1):162-175

12 Sureka B, Yadav K, Garg PK, Khera PS. Moustache sign. Abdom Radiol (NY) 2019;44(4):1629-1630

13 Sureka B, Yadav K, Choudhary GR, Garg PK, Khera PS. Teardrop sign. Abdom Radiol (NY) 2019;44(6):2325-2326

14 Panebianco V, Giganti F, Kitzing YX, et al. An update of pitfalls in prostate mpMRI: a practical approach through the lens of PI-RADS v. 2 guidelines. Insights Imaging 2018;9(1):87-101

15 Ramakrishnan K, Salinas RC. Prostatitis: acute and chronic. Prim Care 2010;37(3):547-563, viii-ix

16 Yuen B, Cho C, Hung E. Pitfalls of prostate imaging reporting and data system Version 2: A pictorial essay. Hong Kong J Radiol 2018;21:280-291

17 Gürses B, Tasdelen N, Yencilek F, et al. Diagnostic utility of DTI in prostate cancer. Eur J Radiol 2011;79(2):172-176

18 Sureka B, Elhence P, Khera PS, et al. Quantitative contrast-enhanced perfusion kinetics in multiparametric MRI in differentiating prostate cancer from chronic prostatitis: results from a pilot study. Br J Radiol 2019;92(1100):20190181

19 Tamada T, Sone T, Jo Y, et al. Prostate cancer: relationships between postbiopsy hemorrhage and tumor detectability at MR diagnosis. Radiology 2008;248(2):531-539

20 Gaur S, Turkbey B. Prostate MR imaging for posttreatment evaluation and recurrence. Radiol Clin North Am 2018;56(2):263-275

21 Grant K, Lindenberg ML, Shebel H, et al. Functional and molecular imaging of localized and recurrent prostate cancer. Eur J Nucl Med Mol Imaging 2013;40(Suppl 1) :S48-S59

22 Klimas R, Bennett B, Gardner WA Jr. Prostatic calculi: a review. Prostate 1985;7(1):91-96

23 Kothari PS, Scardino PT, Ohori M, Kattan MW, Wheeler TM. Incidence, location, and significance of periprostatic and periseminal vesicle lymph nodes in prostate cancer. Am J Surg Pathol 2001;25(11):1429-1432 